\title{
Comparison of two regularization methods for soft $x$-ray tomography at Tore Supra.
}

\author{
A. $\operatorname{Jardin}^{1}$, D. Mazon ${ }^{1}$ and J. Bielecki ${ }^{2}$ \\ ${ }^{1}$ CEA, IRFM, F-13108 Saint Paul-lez-Durance, France. \\ ${ }^{2}$ Institute of Nuclear Physics Polish Academy of Sciences (IFJ PAN), PL-31-342 Krakow, \\ Poland.
}

Corresponding Email: axel.jardin@cea.fr

\begin{abstract}
Soft X-Ray (SXR) emission in the range $0.1 \mathrm{keV}-20 \mathrm{keV}$ is widely used to obtain valuable information on tokamak plasma physics, such as particle transport, magnetic configuration or magnetohydrodynamic (MHD) activity. In particular, 2D tomography is the usual plasma diagnostic to access the local SXR emissivity. The tomographic inversion is traditionally performed from line-integrated measurements of two or more cameras viewing the plasma in a poloidal cross-section, like at Tore Supra (TS). Unfortunately, due to the limited number of measured projections and presence of noise, the tomographic reconstruction of SXR emissivity is a mathematical ill-posed problem. Thus, obtaining reliable results of the tomographic inversion is a very challenging task. In order to perform the reconstruction, inversion algorithms implemented in present tokamaks use a priori information as additional constraints imposed on the plasma SXR emissivity. Among several potential inversion methods, some of them have been identified as well suited to tokamak plasmas. The purpose of this work is to compare two promising inversion methods, i.e. the Minimum Fisher Information (MFI) method already used at TS and planned for WEST configuration, and the alternative $2^{\text {nd }}$ order Phillips-Tikhonov Regularization (PTR) with smoothness constraints imposed on the second derivative norm. Respective accuracy of both reconstruction methods as well as overall robustness and computational time are studied, using several synthetic SXR emissivity profiles. Finally, a real case is studied through tomographic reconstruction from TS SXR database.
\end{abstract}




\section{Introduction}

Soft X-rays (SXR) in the range of $0.1 \mathrm{keV}-20 \mathrm{keV}$ provide valuable information on tokamak plasmas for studying e.g. magnetohydrodynamic (MHD) activity, magnetic equilibrium or impurity transport [1]. In particular tungsten which is widely used as the main plasma facing material on major tokamaks such as ITER, Joint European Torus (JET) or WEST, is a source of concern due to significant radiation losses in the plasma core and thus must be kept under acceptable concentrations [2].

In this context, 2D SXR tomography is a useful tool to observe poloidal asymmetries of impurities and access a rather good estimate of their local concentration. Unfortunately, the local SXR emissivity reconstruction is an ill-posed inverse problem [3] with usually a very limited number of measurements in tokamak plasmas. This limitation is caused by the lack of available space and other technical constraints. Thus to compensate for the lack of experimental data to some extent and to obtain a physically meaningful solution, inversion algorithms implemented in present tokamaks use $a$ priori information as additional constraints imposed on the plasma SXR emissivity. The solution may sensitively depend on the chosen tomographic inversion method [4], thus it is worth to perform a comprehensive benchmark and validate used algorithms.

The purpose of this work is to compare two promising inversion methods, i.e. the Minimum Fisher Information (MFI) method already used at Tore Supra (TS) [5] and planned for WEST configuration, and the alternative $2^{\text {nd }}$ order Phillips-Tikhonov Regularization (PTR) developed at JET for neutron tomography [6] with smoothness constraints imposed on the second derivative norm, see also [7] and more recently [8]. Respective accuracy of both reconstruction methods as well as influence of noise, overall robustness and computational time are studied, using several phantoms of SXR emissivity profiles. Finally, a real case is studied through tomographic reconstruction from TS SXR database. The article is structured as follows. In the next section SXR diagnostic at TS, MFI and PTR tomography methods are presented. The procedure of comparison between the two methods using phantoms of emissivity and results are then detailed in the third section. Finally, conclusion and perspectives are given in the last section.
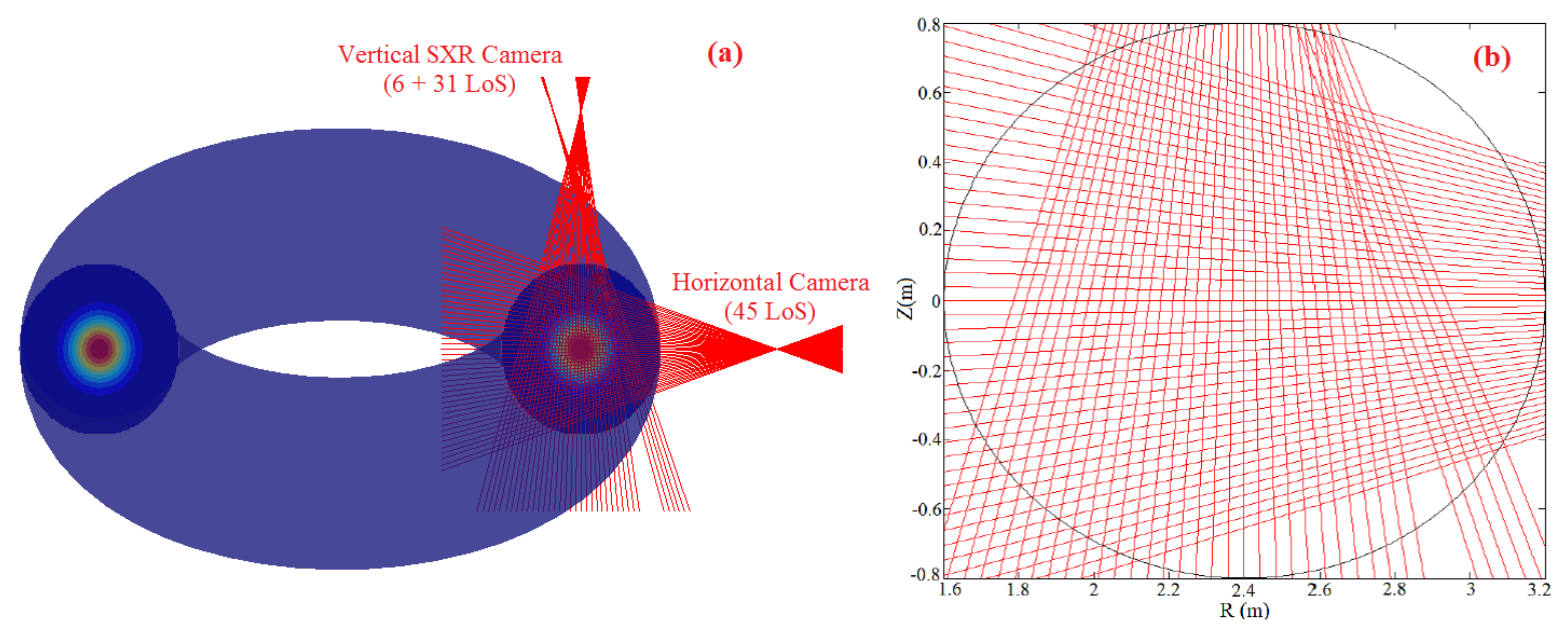

Figure 1. (a) 3D view of SXR detection system on Tore Supra with 2 cameras, 82 Lines of Sight (LoS) in total and (b) 2D poloidal cross-section. 


\section{SXR plasma Tomography at Tore Supra}

\subsection{Generalities}

The SXR diagnostic DTOMOX used at Tore Supra is equipped with two fan-beam cameras. The horizontal one consists of 45 lines of sight $(\operatorname{LoS})$ associated with one pinhole while the vertical camera features $31+6 \mathrm{LoS}$ associated with two pinholes. The geometrical layout of the diagnostic's LoS is shown in figure 1. This configuration enables to obtain the poloidal resolution of $\sim 3 \mathrm{~cm}$ at the toroidal beam width of $\sim 12 \mathrm{~cm}$ in the center of the poloidal crosssection of the vessel. The diagnostic measures line-integrated profiles of the plasma SXR emission between $3-25 \mathrm{keV}$. For this purpose the DTOMOX diagnostic is equipped with a total of $N_{m}=82$ silicon diodes accurately calibrated in their X-ray domain, including the electronics and geometrical correction [9].

The searched SXR emissivity is discretized on a square grid of $1.6 \mathrm{~m} \times 1.6 \mathrm{~m}$ as a matrix of $N_{p} \times N_{p}=N_{p}{ }^{2}$ square elements. Each element is associated to a value $\varepsilon_{j}$ of the SXR emissivity that is assumed to be homogenous within the pixel. It should be noted here that other basis functions than pixels can be used for tomography purposes, see e.g. the use of Fourier-Bessel expansion in [10]. The inverse problem of SXR tomographic reconstruction between emissivity and the line-integrated measurements $m_{i}$ is defined by the set of equations:

$$
m_{i}=\sum_{j} T_{i j} \varepsilon_{j}
$$

where $T_{i j}$ are the transfer matrix coefficients and represent the lengths of the $i$-th chord in the $j$-th pixel in the Line of Sight (LoS) approximation, as defined by collimators geometry with empirical correction coefficients for efficiency of each detector. The residual $\chi^{2}$ between line integrated measurements and reconstructed emissivity is defined as:

$$
\chi^{2}(\boldsymbol{\varepsilon})={ }^{t}(\boldsymbol{m}-T \cdot \boldsymbol{\varepsilon}) \cdot(\boldsymbol{m}-T \cdot \boldsymbol{\varepsilon})
$$

where the superscript $t$ denotes the matrix transpose operation. Simple minimization of $\chi^{2}$ is irrelevant and not applicable in tokamak plasmas due to the ill-conditioned nature of the problem as well as presence of noise in the measured data, with only $N_{m}=82$ projections in TS compared to the amount of information to retrieve $N_{p}{ }^{2} \sim 500-5000$. In this paper, the tomographic inversion will rely on the Tikhonov regularization technique, which consists in adding a priori information on the expected emissivity profile, namely a regularization term $R={ }^{\boldsymbol{t}} \boldsymbol{\varepsilon} H \boldsymbol{\varepsilon}$ which usually imposes smoothness on the gradients of the solution by minimising the functional $\Phi$ :

$$
\boldsymbol{\varepsilon}_{\mathbf{0}}=\underset{\varepsilon}{\operatorname{argmin}} \phi(\varepsilon)=\underset{\varepsilon}{\operatorname{argmin}}\left(\chi^{2}(\varepsilon)+\lambda R\right)
$$

where matrix $H$ is the regularization operator and $\lambda$ denotes the regularization parameter. Thus, the tomographic reconstruction is a compromise between minimization of the residual and regularization of the solution. A vector derivation of equation (3) allows determining the solution $\varepsilon_{0}$ : 


$$
\varepsilon_{0}=\left({ }^{t} T . T+\lambda H\right)^{-1} \cdot{ }^{t} T \cdot \boldsymbol{m}
$$

The regularization parameter $\lambda$ is a free parameter that quantifies the level of "smoothness" of the reconstructed profile. As it will be shown in the section 3.3, $\lambda$ can be determined thanks to an empirical method or by adjusting $\chi^{2}$ according to the experimental noise level, or even via L-curve method. In the following subsections the two methods of SXR emissivity reconstruction: i) based on Phillips-Tikhonov regularization with smoothness constraints imposed on the second derivative and ii) based on the minimization of the Fisher Information, are briefly described.

\subsection{Second order Phillips-Tikhonov Regularization (PTR) method}

The PTR method used here and initially developed at JET for neutron tomography [6] aims at minimizing the curvature $\nabla^{2}$ of the solution, see also [8]. Operator $L=\nabla^{2}$ is a discrete approximation of the Laplacian that imposes a smoothness constraint of the reconstructed solution. In this case, the second-order regularization selects the solution with the least curvature and operator $H_{P T R}$ is:

$$
H_{P T R}={ }^{t} \nabla^{2} \cdot \nabla^{2}
$$

However more advanced constraints on the solution can be applied in a similar way, see e.g. [6]. Additional a priori information that the SXR emissivity should vanish outside the vacuum vessel considered to be at the radial position $r=0.8 \mathrm{~m}$ is included into the reconstruction method. The Generalized Singular Value Decomposition (GSVD) is applied to matrices $T$ and $L$ to calculate the solution:

$$
T=U \cdot \Sigma \cdot X^{-1}, L=V \cdot M \cdot X^{-1}
$$

where $U$ is $N_{m} \times N_{p}{ }^{2}$ orthogonal matrix, $V$ is $N_{p}{ }^{2} \times N_{p}{ }^{2}$ orthogonal matrix, $X$ is $N_{p}{ }^{2} \times N_{p}{ }^{2}$ nonsingular matrix and $\Sigma$ and $M$ are $N_{p}{ }^{2} \times N_{p}{ }^{2}$ diagonal matrices:

$$
\Sigma=\operatorname{diag}\left(\sigma_{1}, \ldots, \sigma_{N p^{2}}\right), M=\operatorname{diag}\left(\mu_{1}, \ldots, \mu_{N p^{2}}\right)
$$

The diagonal entries of $\Sigma$ and $M$ are non-negative and ordered such that:

$$
0 \leq \sigma_{1} \leq \cdots \leq \sigma_{N p^{2}} \leq 1, \quad 1 \geq \mu_{1} \geq \cdots \geq \mu_{N p^{2}} \geq 0
$$

Moreover they are normalized such that:

$$
\left(\sigma_{i}\right)^{2}+\left(\mu_{i}\right)^{2}=1, \text { for } i=1 \ldots N_{P}^{2}
$$

They define the generalized singular values of the matrix pair $(T, L)$ as ratios $\sigma_{i} / \mu_{i}$ (for $i=1 \ldots N_{p}{ }^{2}$ ), that reflect the level of ill-conditioning of the reconstruction problem. In Equation (6) $U$ and $V$ are the matrices of singular vectors of the matrix $T . L^{-1}$.

Then, the regularized solution can be calculated according to the following equation: 


$$
\boldsymbol{\varepsilon}_{\mathbf{0}}=\left\{{ }^{t} T . T+\lambda H_{P T R}\right\}^{-1} \cdot{ }^{t} T \cdot \boldsymbol{m}=\sum_{i=1}^{N_{p}{ }^{2}} \frac{<\boldsymbol{U}_{\boldsymbol{i}}, \boldsymbol{m}>\sigma_{i}}{\sigma_{i}{ }^{2}+\lambda \mu_{i}{ }^{2}} \boldsymbol{X}_{\boldsymbol{i}}
$$

where $\boldsymbol{X}_{\boldsymbol{i}}$ is $i$-th column vector of $X, \boldsymbol{U}_{\boldsymbol{i}}$ is $i$-th column vector of $U$ and $<\ldots>$ denotes the inner product.

\subsection{Minimum Fisher Information (MFI) method}

The SXR tomography method presented here has been developed for TS and a more detailed description can be found in [4], see also [5]. For a given probability distribution $\mathrm{g}(\mathrm{x})$, the associated Fisher information is defined as:

$$
I_{F}(g)=\int \frac{g \prime(x)^{2}}{g(x)} d x
$$

where the prime denotes the derivative with respect to $x$. Considering a discretized inversion problem, it results in the following equation (12) below for the regularization operator $H_{M F I}$ :

$$
H_{M F I}={ }^{t} \nabla \cdot W \cdot \nabla
$$

where $\boldsymbol{\nabla}$ denotes a discrete approximation of the gradient and the ponderation matrix $W$ is defined as:

$$
\begin{cases}W_{i j}=\frac{1}{\varepsilon_{i}} \delta_{i j}, & \varepsilon_{i}>\varepsilon_{\min } \\ W_{i j}=\frac{1}{\varepsilon_{\min }} \delta_{i j}, & \varepsilon_{i}<\varepsilon_{\min }\end{cases}
$$

where $\delta_{i j}$ is Kronecker's delta and $\varepsilon_{\min }>0$ the lower bound used for calculation of the weights. The matrix $W$ imposes flatness at plasma edge where the emissivity is close to zero, and decreases the constraint on the first derivative at plasma core where the emissivity is maximal, for the observation of irregular structures in the core. Similarly to the PTR method, SXR emissivity is constrained to zero outside the vacuum vessel. Since the regularization operator $H_{M F I}$ depends on the unknown emissivity $\varepsilon$, an iterative algorithm is needed to converge to a stable solution, usually in $n \sim 4-6$ steps from a first uniform guess $\varepsilon^{(0)}$. For the $k$-th iteration step $(k \in[1, n])$ :

$$
\left\{\begin{array}{c}
W_{i j}^{(k)}=\min \left(\frac{1}{\varepsilon_{i}^{(k-1)}}, \frac{1}{\varepsilon_{\min }}\right) \delta_{i j} \\
H_{M F I}^{(k)}={ }^{t} \nabla \cdot W^{(k)} \cdot \nabla \\
\boldsymbol{\varepsilon}^{(\boldsymbol{k})}=\left({ }^{t} T \cdot T+\lambda^{(k)}{ }^{t} \boldsymbol{\varepsilon}^{(k-1)} \cdot H_{M F I}^{(k)} \cdot \boldsymbol{\varepsilon}^{(\boldsymbol{k}-\mathbf{1})}\right)^{-1} \cdot{ }^{t} T \cdot \boldsymbol{m}
\end{array}\right.
$$

Any $\varepsilon_{i}^{(k)}<0$ which could appear between two iteration steps is automatically set to zero to avoid unphysical solution. The choice of $\lambda^{(k)}$ will be detailed in section 3.3. The iterative procedure continues until $\boldsymbol{\varepsilon}^{(\boldsymbol{n})} \approx \boldsymbol{\varepsilon}^{(\boldsymbol{n}-\mathbf{1})}$ and $\boldsymbol{\varepsilon}^{(\boldsymbol{n})}$ is taken as the solution for the current time step $t$. For real time tomography, the number of steps can be reduced to 1 - 3 by using the solution at each time step to determine the initial ponderation matrix of the next time step, 
considering that SXR plasma emissivity is slowly evolving compared to the time resolution of the SXR diagnostic.

\section{Benchmarking of the PTR and MFI methods}

\subsection{Procedure of comparison}

The procedure described in this section has been used to compare respective performances of the MFI and PTR tomographic methods. Analytic emissivity phantom models are used to mimic plasma SXR emissivity and then to produce synthetic measurements that will be taken as input in the tomographic inversion algorithms as shown in figure 2. After performing the tomographic reconstruction, consistency between model and reconstruction is checked for both local emissivity and measurements. Such tomographic tests are useful to assess the performances and limits of the algorithms, giving the advantage of knowing the initial emissivity profile in comparison with experimental reconstructions.

Quality of the reconstruction is assessed with the two figures of merits $R M S_{e m}$ and $R M S_{p r}$, which represent the root mean square of the error respectively on the reconstructed emissivity and measurements.

$$
R M S_{e m}=\sqrt{\frac{1}{N_{p}^{2}} \sum_{i}\left(\varepsilon_{i}^{(m o d)}-\varepsilon_{i}^{(r e c)}\right)^{2}}
$$

where $\varepsilon_{i}^{(m o d)}$ denotes the emissivity in the $i$-th element of the model, $\varepsilon_{i}^{(r e c)}$ is the emissivity in the $i$-th element of the reconstruction result, and $N_{p}{ }^{2}$ is the total number of pixels.

$$
R M S_{p r}=\sqrt{\frac{1}{N_{m}} \sum_{i}\left(m_{i}^{(m o d)}-m_{i}^{(r e c)}\right)^{2}}
$$

where $m_{i}^{(m o d)}$ is the signal at the $i$-th detector calculated from the phantom model and $m_{i}^{(r e c)}=\sum_{j} T_{i j} \varepsilon_{j}^{(r e c)}$ is the signal at the $i$-th detector based on the reconstructed emissivity. $N_{m}=82$ is the number of measurements (LoS).

It should be noted that discretization of the emissivity profile in a limited number of grid points induces a discrepancy between the resulting synthetic measurements and what should be expected from a continuous emissivity profile, in particular at low spatial resolutions. In order to avoid such discretization issues, emissivity profiles have been systematically linearly interpolated on a fixed grid $N_{p}{ }^{2}=100 \times 100$ (high resolution for TS plasmas) for the production of synthetic measurements and comparison at different spatial resolutions $N_{p}$.

\subsection{Phantom models of SXR emissivity}

Three different sets of phantom models are used to mimic various experimental cases such as impurity injections or poloidal asymmetries. One example for each model - Gaussian, hollow, and banana shape - is presented in figure 2. The simple Gaussian model is given by the following formula: 


$$
\Im(R, Z)=\exp \left(-\frac{(R-\Delta R)^{2}}{2 \sigma^{2}}-\frac{(Z-\Delta Z)^{2}}{2 \sigma^{2}}\right)
$$

where $(\Delta R, \Delta Z)$ are the plasma center coordinates and $\sigma$ represents the standard deviation of the Gaussian. The hollow model is obtained by subtracting two Gaussian phantoms with the same emissivity center but different variances $\sigma_{1}^{2}>\sigma_{2}^{2}$ :

$$
\mathcal{H}(R, Z)=\mathfrak{S}_{1}(R, Z)-\mathfrak{S}_{2}(R, Z)
$$

Then, the banana model is derived from the hollow model with introduction of a Low-Field Side (LFS) or High-Field Side (HFS) poloidal asymmetry as follows:

$$
\mathcal{B}(R, Z)=\mathcal{H}(R, Z) * \exp \left(-\frac{\left(R-\Delta R_{\text {asym }}\right)^{2}}{2 \sigma_{\text {asym }}^{2}}-\frac{\left(Z-\Delta Z_{\text {asym }}\right)^{2}}{2 \sigma_{\text {asym }}{ }^{2}}\right)
$$

where the point $\left(\Delta R_{\text {asym }}, \Delta Z_{\text {asym }}\right)$ denotes the center of the asymmetry chosen on the corona of the corresponding hollow profile, and $\sigma_{\text {asym }}$ represents the asymmetry spatial extent. Emissivity profiles are normalized such that RMS equal to one represents $100 \%$ of global reconstruction error and zero represents a perfect reconstruction.
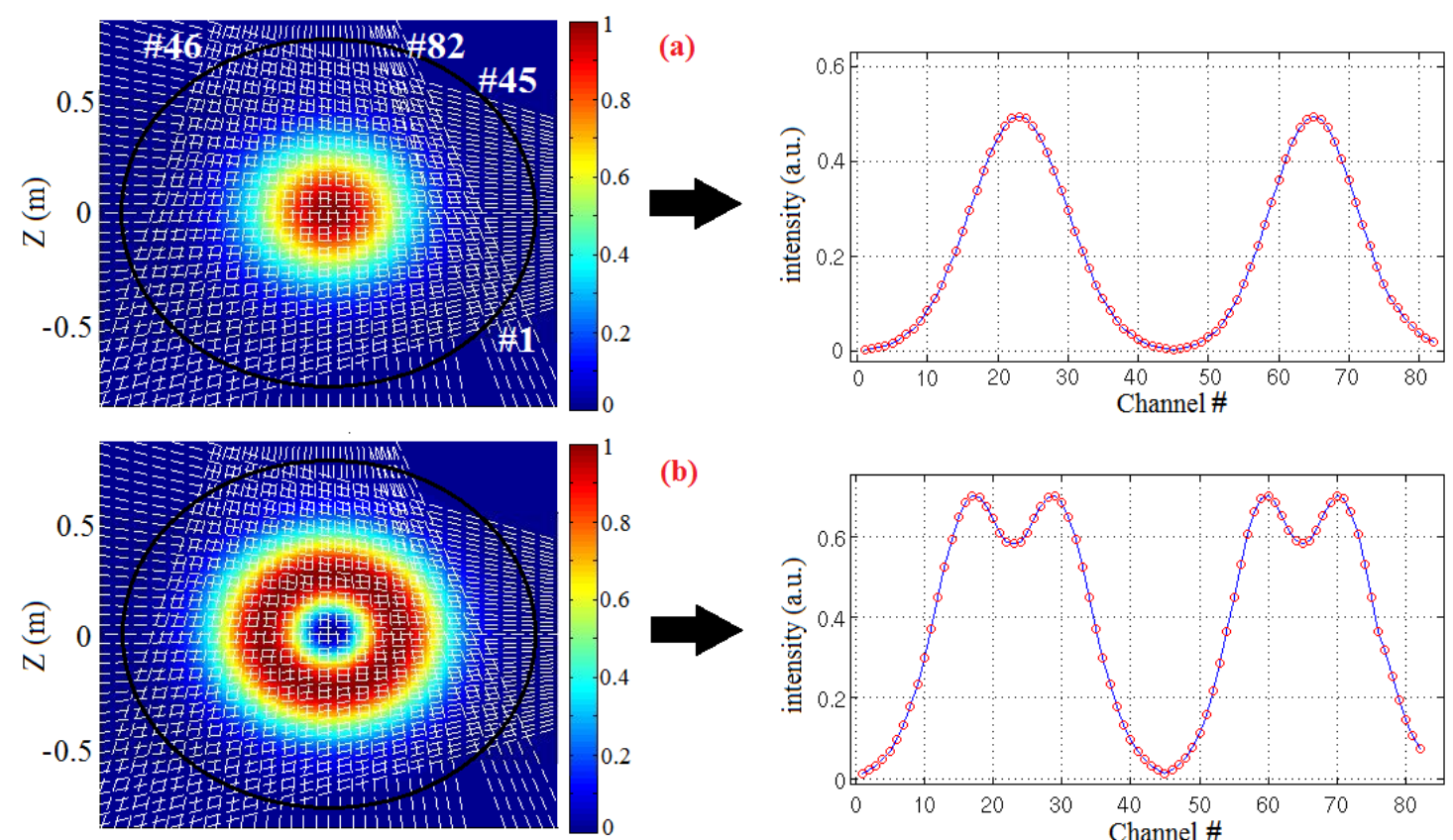

(b)
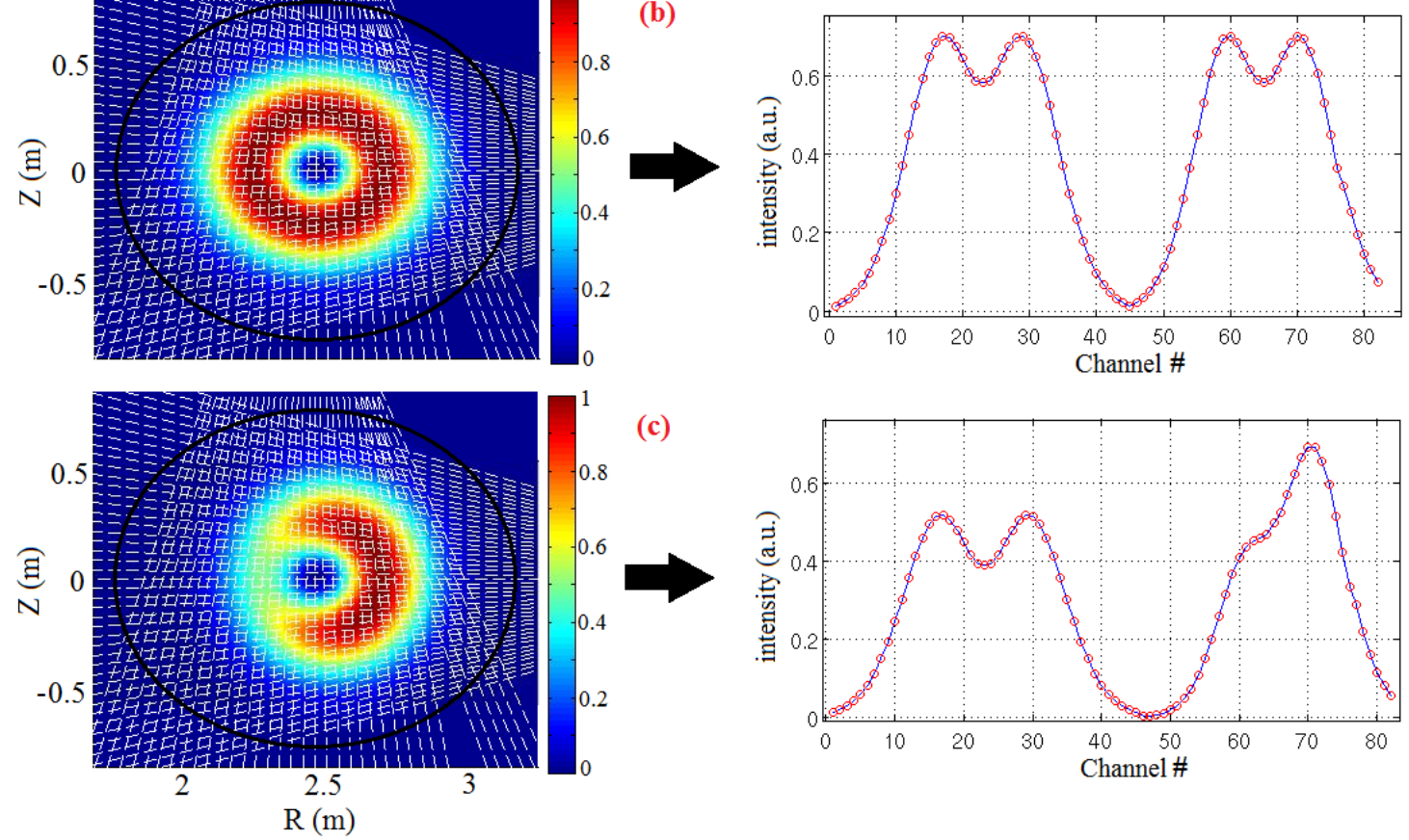

(c)

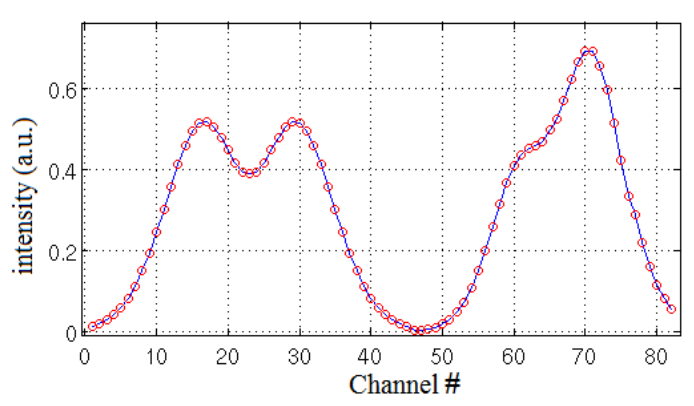

Figure 2. SXR emissivity phantoms and associated TS synthetic measurements for (a) Gaussian (b) hollow and (c) LFS banana models. The black curve denotes the vacuum vessel limit and LoS are indicated in dashed white $\underline{\text { lines. }}$ 


\subsection{Choice of the regularization parameter}

As mentioned in section 2.1, the regularization parameter $\lambda$ quantifies the level of "smoothness" of the reconstructed profile and corresponds to a balance between overfitting of measurements and oversmoothing of the solution. For the PTR method $\lambda$ is selected using L-curve method [11]. The method is based on a plot, for all valid regularization parameters, of the magnitude of the regularized solution versus the magnitude of the corresponding residual. The L-curve when plotted in log-log scale has usually a characteristic L-shaped appearance with a distinct corner separating the vertical and horizontal parts of the curve. The idea is schematically illustrated in figure 3(a). In this way, the L-curve displays the optimum of the minimization of these two quantities, which is the key of the regularization method. The optimal choice of the regularization parameter corresponds to the L-curve's corner. An application of the method for selection of the optimal regularization parameter for a Gaussian emissivity model reconstruction is shown in figure $3(b)$.
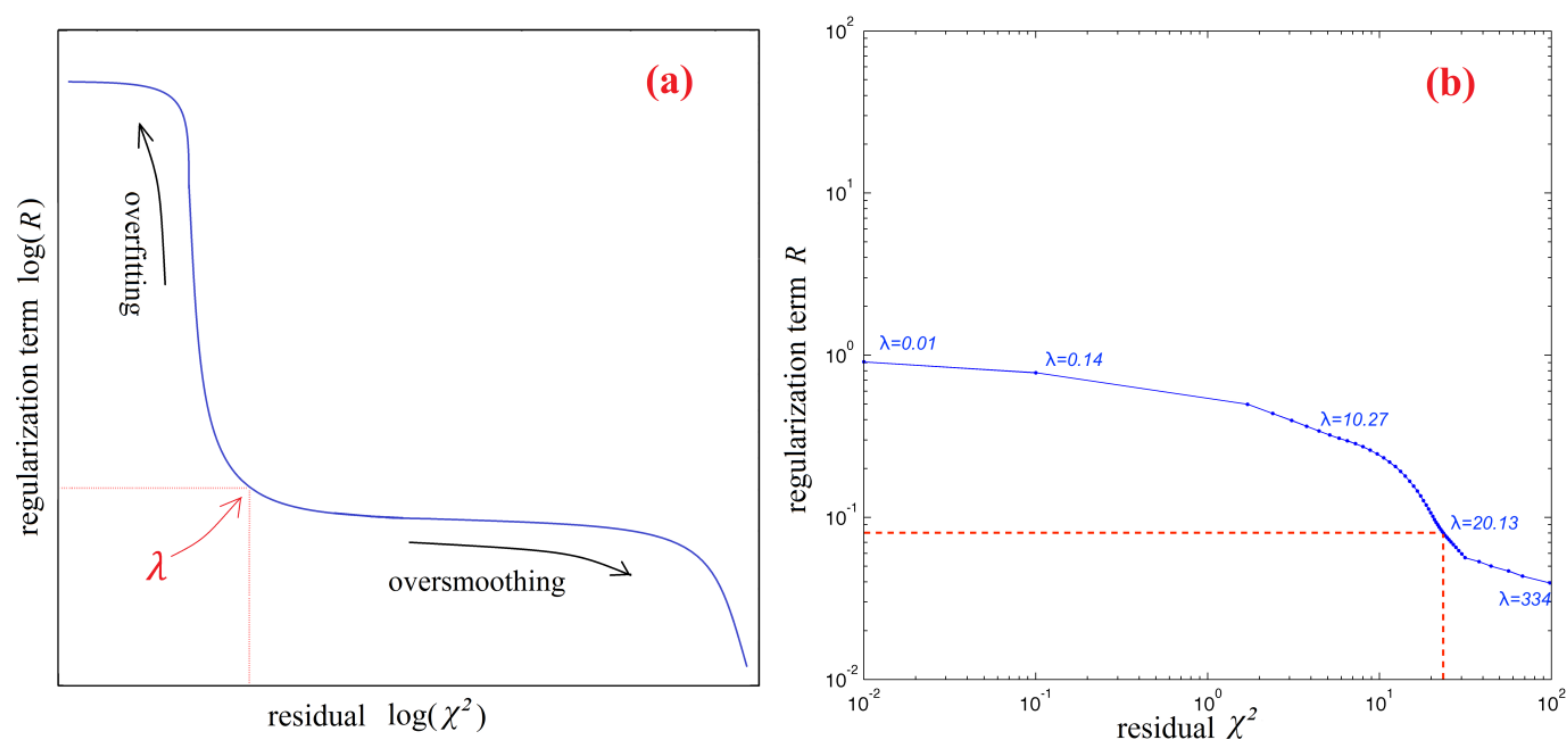

Figure 3. Illustration of the L-curve corner method for (a) an ideal L-curve shape and (b) a Gaussian phantom emissivity model (PTR method) with $\sigma=0.2 \mathrm{~m}$. Optimal value of regularization parameter $\lambda$ is a balance between overfitting of measurements and oversmoothing of the solution.

For the MFI method $\lambda^{(k)}$ (see equation (14)) is determined at each iteration step using a fast empirical method on the matrix traces which has given satisfactory results so far:

$$
\lambda^{(k)}=\frac{\operatorname{Tr}\left({ }^{t} T . T\right)}{\operatorname{Tr}\left(H_{M F I}^{(k)}\right)}
$$

A dynamic regula-falsi method has also been recently implemented according to the work performed in [12]. In this case the regularization parameter is optimized such that smoothness of solution matches the noise level in measurements. This is achieved by defining a new residual:

$$
\chi_{N}^{2}=\frac{1}{N_{m}} \sum_{i} \frac{\left(m_{i}-m_{i}^{(r e c)}\right)^{2}}{\sigma_{i}^{2}} \approx 1
$$


where $\sigma_{i}^{2}$ denotes the variance of the expected noise level on the $i$-th channel. Tolerance on $\chi_{N}^{2}$ convergence to 1 is set to $5 \%$. Figure 4 shows the evolution of the residual in blue and the regularization term in red as a function of the regularization parameter $\lambda$ for a Gaussian phantom emissivity model with $\sigma=0.2 \mathrm{~m}$ and $N_{p}=50$ (a) without and (b) with addition of $10 \%$ zero-mean white Gaussian noise in each channel. Corresponding emissivity reconstruction error $R M S_{e m}$ is plotted in green. The second definition of the residual from equation (21) is only used for non-zero characterized noise level. Green squares denote $\lambda$ optimization for both traces ratio and regula-falsi methods. As a result, the empirical traces ratio method is satisfying in terms of $R M S_{\text {em }}$ in absence of noise but tends to overfit measurements, while the second method is more suitable in case of known non-zero noise level. Experimental noise level in SXR measurements on Tore Supra is usually about a few percent. In the following sections zero noise level has been assumed in synthetic measurements for simplicity, thus the traces ratio method is used for $\lambda$ computation (MFI method). The PTR method uses the L-curve's corner method as presented in figure 3. Further details about robustness of the PTR method against the noise level can be found in [6].
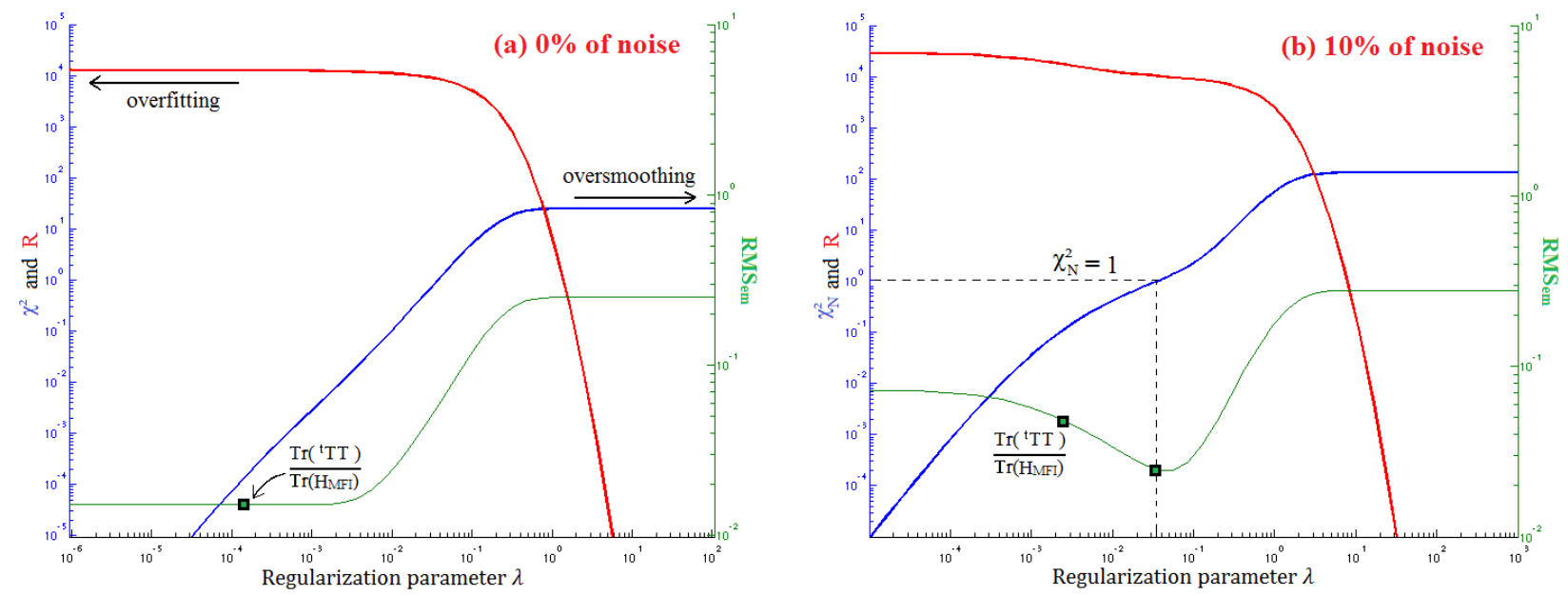

Figure 4. Application of regula-falsi and traces ratio method on a Gaussian phantom emissivity model with $\sigma=$

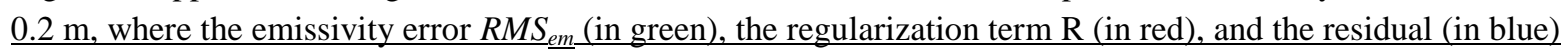
(a) $\chi^{2}$ without noise and (b) $\chi_{N}^{2}$ with $10 \%$ zero-mean white Gaussian noise are plotted against the regularization parameter $\lambda$ (MFI method).

\subsection{Results}

The results of the tomographic reconstruction test are presented in figure 5 for each of the 3 phantom models and both PTR and MFI methods. While the reconstruction of the Gaussian shape emissivity profile in figure 5(a) gives very satisfactory results, reconstructions of less regular shapes like corona emissivity in figure 5(b) or LFS asymmetry in figure 5(c) are not perfect due to the limited number of projections ( 2 cameras with $82 \mathrm{LoS}$ in total). In fact, information tends to concentrate into poles of emissivity defined by the geometry of the LoS. Nevertheless, global features like corona or LFS asymmetry are still clearly identifiable. 

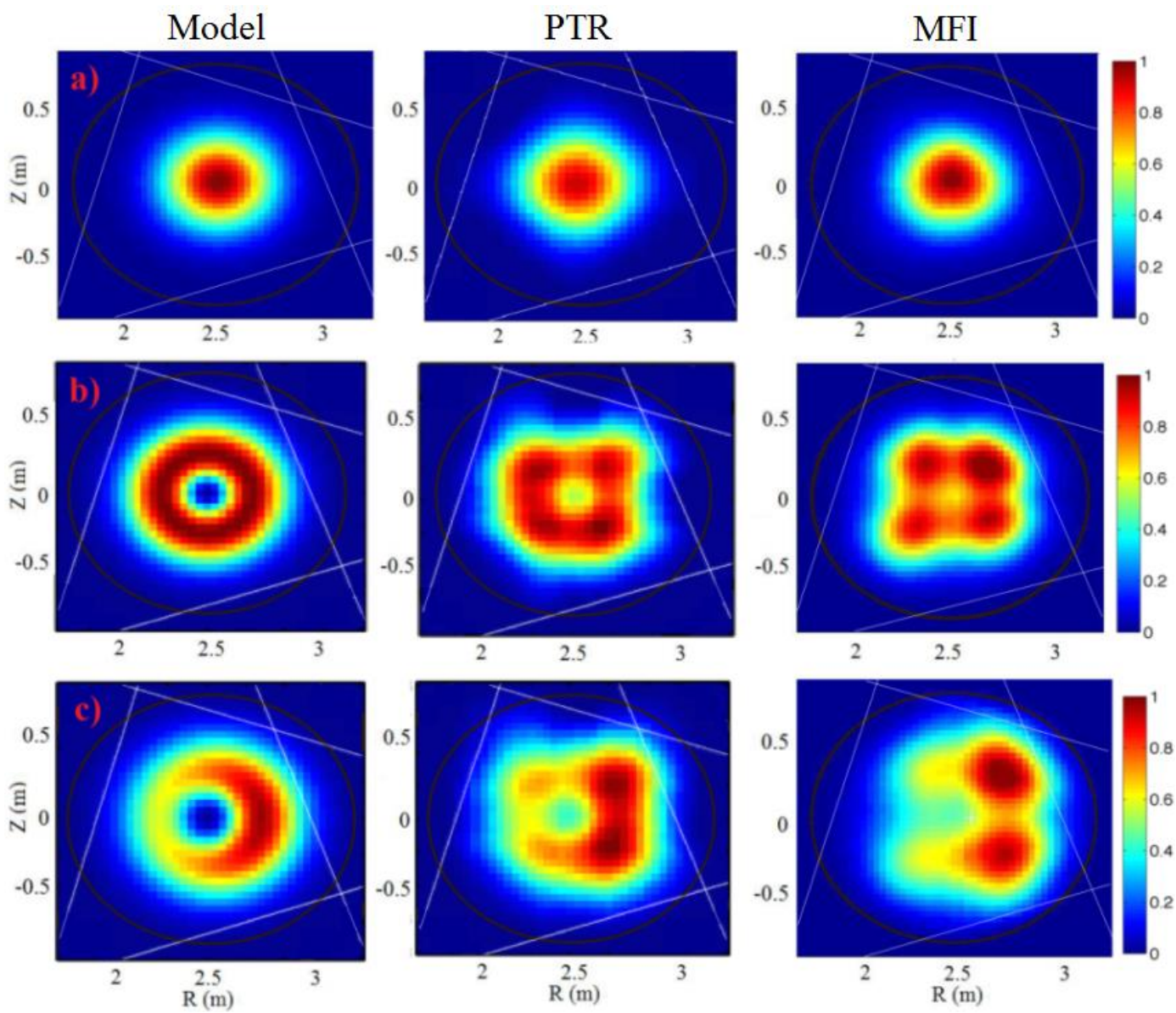

Figure 5. Tomographic reconstruction tests with PTR and MFI methods for (a) Gaussian, (b) Hollow and (c) LFS Banana centered emissivity phantom models with $\sigma=0.2 \mathrm{~m}$. Vacuum vessel is plotted in black and edge LoS are indicated in white.

Quality of reconstruction versus plasma emissivity extent $\sigma$. Here tomographic reconstruction capacities are studied for regular centered Gaussian phantom models with a scan of the standard deviation $\sigma$ from $0.1 \mathrm{~m}$ to $0.3 \mathrm{~m}$, see figure 6 . Values of $\sigma$ greater than 0.3 $\mathrm{m}$ are not considered as relevant with respect to the available plasma volume inside the vacuum vessel. The grid size was arbitrary fixed to $N_{p}=40$, see the next paragraph for the effect of the spatial discretization on the reconstruction. The usual range of experimental SXR emissivity extent observed on TS is also indicated in black. It can be seen that for TS plasmas both MFI and PTR methods are satisfying and give similar results with $R M S_{e m} \sim 1 \%$, with a slightly better quality for the MFI method in the TS range. This trend seems to reverse for higher $\sigma$ values, which can be explained by the different regularization operators of the two methods. Indeed, the PTR method simply minimizes the curvature (second derivative) of the solution while in the MFI method the ponderation matrix $W$ flattens the gradient at the plasma edge. This can degrade the MFI reconstructions for high SXR emissivity extents with respect to the considered plasma volume, where the SXR emissivity level is still substantial at the very plasma edge. Nevertheless, it should be noted that such situations with high emissivity extent were not encountered experimentally so far. 

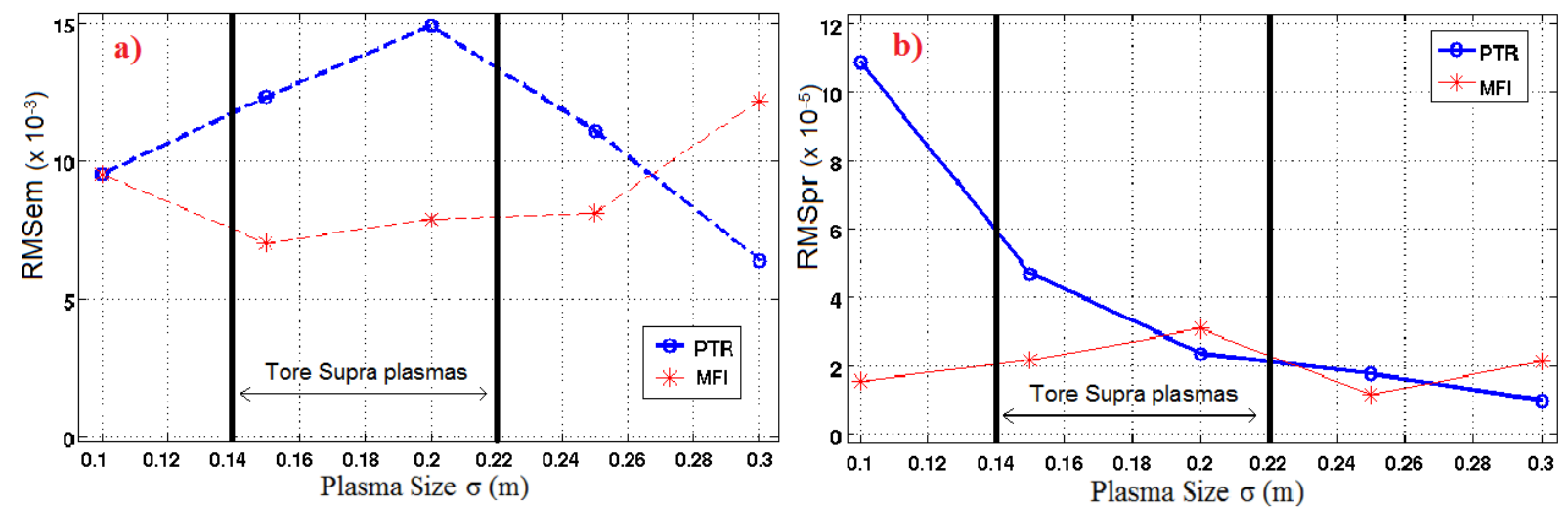

Figure 6. Quality of reconstruction versus emissivity extent $\sigma$ for the PTR and MFI methods and the Gaussian phantom model with (a) error on the emissivity $R M S_{e m}$ and (b) error on the projections $R M S_{p r}$ -

Quality of the reconstruction versus the grid size $N_{p}$. In figure 7 the two regularization processes are compared using a set of 108 phantoms per grid point, including the 3 plasma models for different plasma sizes from $\sigma=0.15 \mathrm{~m}$ to $0.25 \mathrm{~m}$ and for different positions $(\Delta R$, $\Delta Z$ ) from $-0.2 \mathrm{~m}$ to $+0.2 \mathrm{~m}$ around the vessel center, in order to cover all the possibilities inside the available plasma volume. As a general trend, it is clearly seen that a finer grid leads to better reconstructions, see figures 7(a) and 7(b), but with an extra cost of the computational time per inversion, see figure 7(c). Different but comparable computing units were used for the calculations: a standard core i5 laptop for the PTR method and a core i7 laptop for the MFI method. Grids finer than $N_{p}{ }^{2}=50 \times 50$ do not give better reconstructions than $R M S_{\text {em }} \sim 2$ $5 \%$ while the computational time is an increasing exponential function of $N_{p}$. It is found that the PTR method is globally slightly more accurate in terms of $R M S_{e m}$ while the MFI method is 1 to 2 orders of magnitude faster but tends to overfit measurements, see figure 7(b), due to the $\lambda$ optimization method described in section 3.3. For the PTR method most of the computational time is spent in GSVD applied to $T$ and $L$ matrices. This time was taken into account for each reconstruction. This approach enables to assess the more general case when matrix $L$ is time dependent (e.g. depends on magnetic configuration as presented in [6]). In such a case $L$ has to be computed individually for every time interval of interest in the plasma discharge. For the MFI method the computational time is $\sim 10 \mathrm{~ms}-100 \mathrm{~ms}$ per inversion for $20<N_{p}<30$, useful for fast and robust inversions, and $\sim 1 \mathrm{~s}$ per inversion for finer analysis at $N_{p}=50$. These results remain in agreement with previous results in [5].
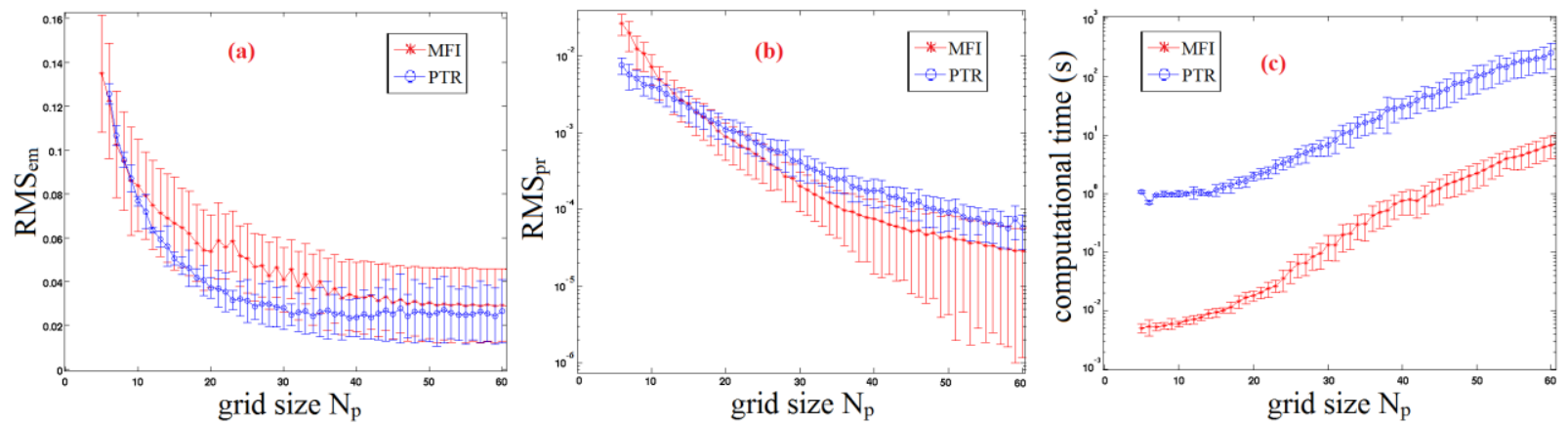

Figure 7. Quality of reconstruction versus grid size for the PTR and MFI methods applied to a set of phantom $\underline{\text { emissivity models with (a) error on the emissivity } R M S_{\underline{e m}}}$ (b) error on the projections $R M S_{\underline{p r}} \underline{\text { and (c) associated }}$ computational time per tomographic inversion. 
HFS asymmetry on TS shot \#46564 with tungsten (W) Laser Blow-off (LBO). As seen above in this section, due to the low number of projections tomographic inversions do not give perfect reconstructions and poles of emissivity appear for non-regular emissivity shapes. A HFS asymmetry was observed with DTOMOX in TS\#46564 during W LBO with two local emissivity maxima on the HFS, which is reprocessed here with the MFI method and presented in figure 8. The obtained results are in good agreement with [5]. The increase in SXR emissivity during the LBO time interval indicated in figure 8(a) is attributed to W radiation, thus background is subtracted from the SXR signal in figure 8(b) and resulting tomographic inversion is presented in figure 8(c). A HFS banana phantom model in figure 8(d), for which synthetic measurements fit rather well to the experimental ones in figure $8(\mathrm{~b})$, has been used to investigate if the presence of a HFS asymmetry was consistent with the experimental tomogram. The experimental reconstruction in figure $8(\mathrm{c})$ is thus compared with the reconstructed emissivity profile shown in figure 8(f) obtained from the phantom model, figure 8(d). As a result, the two tomograms show a similar pattern of poles of emissivity in terms of both position and intensity, validating the presence of an experimental HFS asymmetry in W radiation.
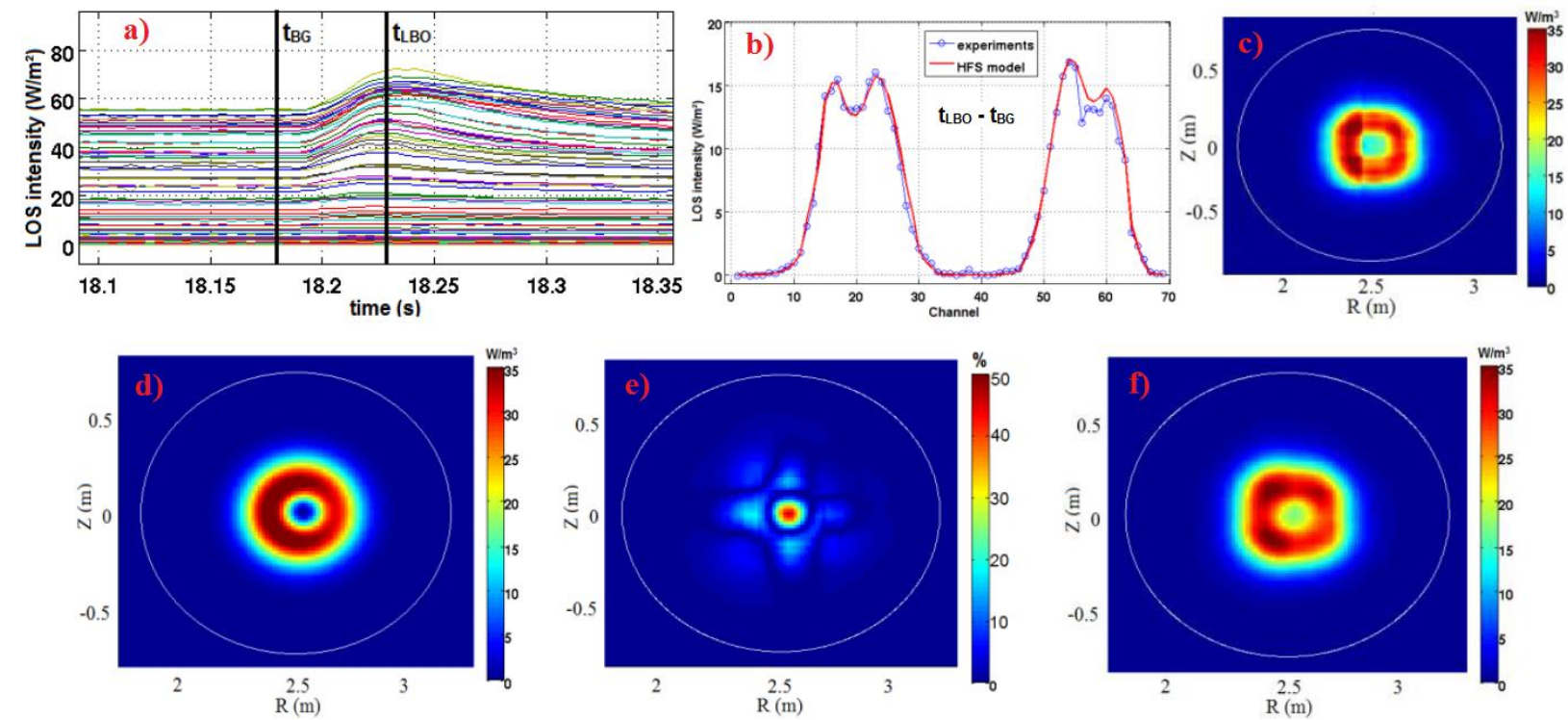

Figure 8. W LBO during TS shot \#46564 with (a) time evolution of the SXR measurements, (b) background-

subtracted measurements taken at $t_{L B O}$ and respective HFS banana model, (c) experimental tomographic reconstruction, (d) HFS banana emissivity model and associated (e) reconstruction error on emissivity and (f) emissivity reconstructed from the model.

\section{Conclusion and perspectives for WEST}

In this paper, the $2^{\text {nd }}$ order Phillips-Tikhonov Regularization and the Minimum Fisher Information method for SXR plasma tomography at Tore Supra have been compared. The two inversion methods are both found to be suitable for TS plasma tomography and the benchmarking between the two methods is encouraging. The importance of the choice of the regularization parameter and of the discretization of the plasma domain has been highlighted. The benchmarking results show that the MFI method is one to two orders of magnitude faster 
while the presented PTR method is overall slightly more accurate. The global accuracy of the tomographic reconstructions is around $1-5 \%$, with best reconstructions reached for regular Gaussian emissivity shapes. Both, robust and fast inversions $\sim 10^{-3}$ s at $N_{p} \sim 30$ in the prospect of real time control with faster computing unit such as FPGAs, as well as longer but finer analysis $\sim 10^{-2} \mathrm{~s}-1 \mathrm{~s}$ at $N_{p} \sim 40-50$ are feasible.

It has also been shown with phantom models that perfect tomograms cannot be obtained due to the low number of projections, especially for emissivity profiles diverging from a regular Gaussian shape. Nevertheless, the physical information on SXR emissivity is still present on tomograms and gives useful information on poloidal asymmetries and impurity radiation. Thus, tomographic reconstructions will be valuable for impurity transport studies (tungsten in particular) on WEST. The SXR diagnostic of WEST will be composed of two GEM cameras [13] with a tunable spectral response and installed in the same poloidal cross-section as shown in figure 9. The plasma coverage will be ensured by a total of $200 \mathrm{LoS}$ with a spatial resolution $<1 \mathrm{~cm}$ at the plasma core, to be compared with $\sim 3 \mathrm{~cm}$ for TS diodes, and with a smaller plasma volume due to the new divertor configuration. Thus, a global better spatial resolution is expected in WEST than in TS for SXR plasma tomography.

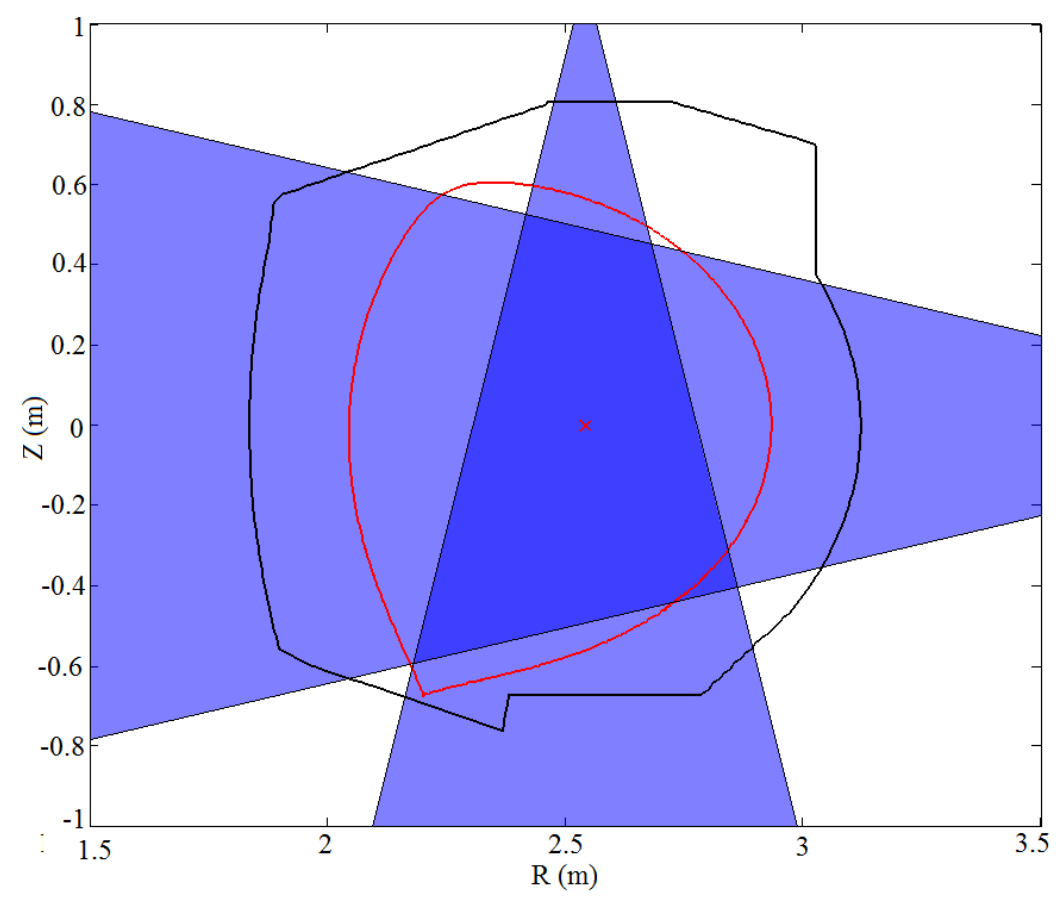

Figure 9. SXR diagnostic geometry planned for WEST represented in a 2D poloidal cross-section, with a total of $\sim 200 \mathrm{LoS}$ (in blue). The black line represents the new chamber wall in divertor configuration. Last-closed flux surface and magnetic axis are plotted in red. 


\section{Acknowledgement}

This work has been carried out within the framework of the EUROfusion Consortium. The views and opinions expressed herein do not necessarily reflect those of the European Commission.

\section{References}

[1] D. Vezinet et al., Impurity density derivation from bandpass soft x-ray tomography: applicability, perspectives and limitations, Nucl. Fusion 54 (2014) 083011

[2] T. Pütterich et al., Calculation and experimental test of the cooling factor of tungsten, Nucl. Fusion 50 (2010) 025012

[3] L.C. Ingesson et al., Soft X ray tomography during ELMs and impurity injection in JET, Nucl. Fusion 38 (1998) 1675.

[4] M. Anton et al., X-ray tomography on the TCV tokamak, Plasma Phys. Control. Fusion 38 (1996) 1849.

[5] D. Mazon et al., Soft x-ray tomography for real-time applications: present status at Tore Supra and possible future developments, Review of Scientific Instruments 83 (2012) 063505.

[6] J. Bielecki et al., Phillips-Tikhonov regularization with a priori information for neutron emission tomographic reconstruction on Joint European Torus, Rev Sci Instrum. (2015) 86(9):093505

[7] S.H. Lee et al., Modified Phillips-Tikhonov regularization for plasma tomography, Current Applied Physics 10 (2010) 893.

[8] A. Wingen et al., Regularization of soft-X-ray imaging in the DIII-D tokamak, Journal of Computational Physics 289 (2015) 83.

[9] D. Mazon et al., Soft X-ray tomography optimization on Tore Supra for real time applications, Review of Scientific Instruments 79 (2008) 10E321.

[10] P. Franz et al., Soft X ray tomographic imaging in the RFX reversed field pinch, Nucl. Fusion, Vol. 41, No. 6 (2001) 695.

[11] P.C. Hansen, Analysis of discrete ill-posed problems by means of the L-curve, SIAM Rev. 34 (4), (1992) 561-580.

[12] J. Mlynar et al., Tikhonov regularization adapted to the real-time tomography, 1st TM IAEA on Fusion Data Processing, Validation and Analysis (2015)

[13] D. Mazon et al., Design of soft X-ray tomographic system in WEST using GEM detectors, Fus. Eng. Design 96-97 (2015) 856-860 